\title{
Dynamic control of kinematically redundant robotic manipulators
}

\author{
ERLING LUNDE, OLAV EGELAND and JENS G. BALCHEN $\dagger$
}

Keywords: Robots, Kinematically redundant manipulators, Non-linear control, Optimal control.

Several methods for task space control of kinematically redundant manipulators have been proposed in the literature. Most of these methods are based on a kinematic analysis of the manipulator. In this paper we propose a control algorithm in which we are especially concerned with the manipulator dynamics. The algorithm is particularly well suited for the class of redundant manipulators consisting of a relatively small manipulator mounted on a larger positioning part.

The main idea behind the algorithm is to augment the task space position vector by a set of generalized coordinates for the positioning part, so that the augmented task space position vector constitutes a set of generalized coordinates for the manipulator. We then show how to choose a proper reference for the positioning part. Feeback linearization and decoupling in the augmented task space make it straightforward to develop a controller which distributes the high frequency motion to the small manipulator and the low frequency motion to the positioning part.

In a simulation experiment, this control algorithm performed significantly better than controllers using generalized inverses of the manipulator Jacobian.

\section{Introduction}

Traditionally, robotic manipulators have been designed with a minimum number of joints - to reduce the complexity of the control system - and with rather large and heavy links - to ensure good positioning accuracy through the stiffness of the arm.

Today, we can avoid these clumsy mechanisms by the use of advanced control theory and high speed computers. Lightweight constructions with kinematic redundancy can be controlled through high speed motion with good positioning accuracy and moderate power consumption.

This paper considers a redundant manipulator consisting of a small, fast (nonredundant) manipulator mounted on a large, slow positioning part. Here we take advantage of the redundancy to design a system with a large working area, high bandwidth and moderate power consumption. The idea is to distribute the fast motion to the small manipulator and the slow, gross motion to the positioning part.

Both the manipulator structure and the control system are inspired by the human arm (Fig. 1). The upper arm and the forearm (and the body itself) can be considered to constitute the positioning part, and the hand represents the small, fast manipulator. This is easily illustrated by observing the process of handwriting. The human arm has a large number of degrees of freedom: the shoulder is a 3 dof. joint,

Received 3 March 1987.

$\dagger$ The Norwegian Institute of Technology, Division of Engineering Cybernetics, N-7034 Trondheim, Norway, 


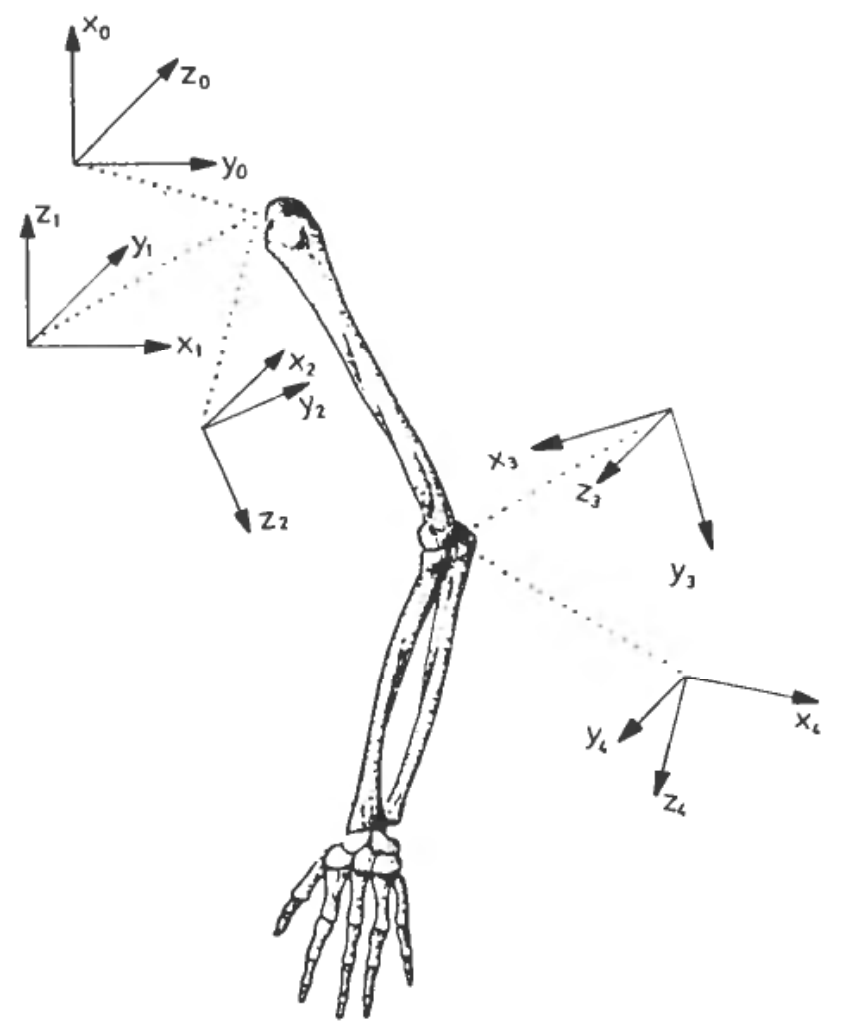

Figure 1. Human arm (the rotational axes are denoted $z_{1} \cdots z_{5}$ ).

the elbow is a 2 dof. joint and the wrist and the hand have got a very large number of joints and links.

The same principle applies to a manipulator mounted on a free swimming ROV. Here, the submarine itself corresponds to the positioning part.

Redundant manipulators are also useful when there are obstacles in the working area. In this case, redundant degrees of freedom may be required to position the end effector without colliding with the obstacles (Khatib 1985).

\subsection{Previous work}

Industrial manipulator systems have usually been programmed by a teach-in procedure where the manipulator arm is led through the actual task by a human operator. The joint displacements are sampled and recorded, and later used as a servo reference when copying the operations. The tracking is then done by using a PID controller for each servo loop.

In many applications, the reference trajectory for the manipulator is specified in terms of task coordinates which are the position and orientation of the end effector in a task-defined coordinate system. The conventional way of controlling a manipulator when the reference trajectory is given in the task space, is to transform the task space reference to the joint space. A controller is then designed to track this refer- 
ence with a PID controller at each joint (Luh 1983) or by means of the computed torque technique (Bejczy 1974).

This solution cannot be applied to redundant manipulators, as there is no unique position transformation from the task space to the joint space for this type of manipulator. In most previous work on redundant manipulators, this problem has been solved by using the manipulator Jacobian to transform the task space velocity or acceleration to the joint space. Since the Jacobian matrix of a redundant manipulator is rectangular and not invertible, generalized inverses of the Jacobian have been used (Klein and Huang 1983) and (Klein and Chirco 1987). By using an appropriate generalized inverse, a performance index that is quadratic in joint velocities is minimized. However, when this technique is used, the manipulator may reach singularities or lose degrees of freedom when the joints reach their limit (Baillieul 1985). A vector in the null space of the Jacobian has been added to the joint velocity to improve the result that is obtained from using generalized inverses. This null vector has been used to avoid singularities (Yoshikawa 1985) and minimize actuator torques (Hollerbach and Suh 1985). The use of generalized inverses is a purely kinematic approach which does not take the dynamic properties of the redundant manipulator into account. Because of this, a well coordinated use of control variables or small control deviations is not necessarily the result.

Salisbury and Abramowitz (1985) discussed redundant manipulators with a small, fast manipulator on a positioning part, and investigated a very simple mechanism of this kind. A planar two-link mechanism with rotary joints is used to position the end of link 2 in one direction. Here the motion of the mechanism is divided into external motion and internal or null motion in order to obtain a high bandwidth with moderate power consumption. The external motion is controlled with a high bandwidth, while the internal motion which maintains a desirable configuration has a lower bandwidth.

In a recent work by Egeland (1987) the control algorithm proposed in this paper was successfully implemented on an industrial manipulator system. Experiments were carried out on the 8 degrees of freedom Trallfa TRACS spray painting robot.

The proposed control system has previously been presented in Lunde, Egeland and Balchen (1985).

\subsection{Outline of the paper}

We consider the control of a particular class of redundant manipulators, i.e. a small non-redundant manipulator mounted on a positioning part.

A control system which is particularly suited for this class of manipulators is presented. In this control system the task space position vector is augmented in such a way that the augmented task space position vector contains a set of generalized coordinates of the redundant manipulator. Singularities and the loss of degrees of freedom are avoided by specifying a suitable augmented task space reference. Advantage is taken of the good dynamic characteristics of this class of manipulators by using feedback linearization and decoupling in the augmented task space.

The control system is then applied to a planar four-link redundant manipulator, and the performance of the control system is compared to the performance of controllers using a generalized inverse of the manipulator Jacobian. 


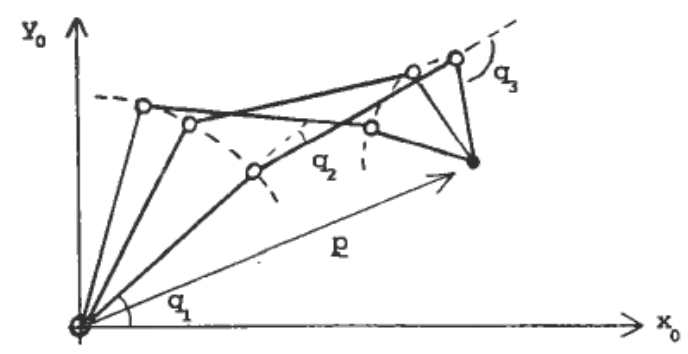

Figure 2. Redundant manipulator.

\section{Basic definitions}

The dynamic model of a robotic manipulator is multi-variable, non-linear and highly coupled. Similarly, the kinematic model can also be complex, especially in the case of redundant degrees of freedom.

\subsection{Dynamics}

The dynamic equation for a manipulator is usually written (Luh, Walker and Paul 1980)

$$
M(q) \ddot{q}=\boldsymbol{n}(\boldsymbol{q}, \dot{\boldsymbol{q}})+\boldsymbol{\tau}
$$

where $q$ is the vector of the $n$ joint displacement and $\tau$ is the vector of $n$ generalized forces. $M(q)$ is the inertia matrix. The vector $\boldsymbol{n}(\boldsymbol{q}, \dot{\boldsymbol{q}})$ contains terms due to Coriolis, centrifugal, gravitational and viscous friction forces.

\subsection{Kinematics}

The relation between the joint space coordinates $q$ and the task space position $p$ is in general non-lineart

$$
\boldsymbol{p}=\boldsymbol{h}(\boldsymbol{q})
$$

where $\operatorname{dim} q=n$ and $\operatorname{dim} p=m$.

The inverse transformation

$$
q=h^{-1}(p)
$$

will exist only in the non-redundant case, i.e. when $n=m$, and-because of its complexity - it will be practical to solve only when $n$ is small. For a kinematically redundant manipulator $(n>m)$ the system (3) will be underdetermined. This means that given a task space position $p$, there are a corresponding infinite-number of joint space positions $q$. This is illustrated in Fig. 2 for a simple planar manipulator, where we consider only the $x y$-position of the tip of the manipulator arm.

The velocity transformation can be written

$$
\dot{\boldsymbol{p}}=\boldsymbol{J}(\boldsymbol{q}) \dot{\boldsymbol{q}}
$$

$\dagger$ In this paper we consider the task space position vector $p$ to represent both the position and the orientation of the end effector. 
where the Jacobian matrix is defined by

$$
J(\boldsymbol{q})=\frac{\partial \boldsymbol{p}}{\partial \boldsymbol{q}}
$$

The inverse velocity transformation will be well-defined only when the Jacobian matrix is square $(n=m)$ and non-singular, i.e. $J^{-1}(q)$ exists.

\subsection{Control}

Accurate and fast manipulator control is not straightforward because of the very complex nature of the dynamics (1). This means that calculating a proper control $\tau$ will be time-consuming, even on a very fast computer.

Most tasks are most naturally defined in the task space. Hence, using redundant manipulators, the tracking of a task space reference $\boldsymbol{r}$ also represents a considerable problem as we need to transform the reference itself, the control deviation $(\boldsymbol{r}-\boldsymbol{p})$, or some task space control vector into the joint space, in which the real actuators are controlled.

\section{Feedback linearization and decoupling. Review.}

By choosing a proper control vector $\tau$, the model (1) can be transformed into a system of $n$ decoupled double integrators. This wellknown technique has previously been used by several authors, and is known by different names such as 'Resolved Acceleration Control' (Luh et al. 1980) and 'The Computed Torque Technique' (Bejczy 1974).

We define a joint space state vector $z=\left[z_{1}^{T}, z_{2}^{T}\right]^{T}$ where $z_{1}=q$ and $z_{2}=\dot{q}$. The control vector

$$
\boldsymbol{\tau}=M(\boldsymbol{q}) \boldsymbol{u}-\boldsymbol{n}(\boldsymbol{q}, \dot{\boldsymbol{q}})
$$

applied to the model (1) gives the system of $n$ decoupled double integrators in joint space

$$
\ddot{\boldsymbol{q}}=\boldsymbol{u}
$$

where $\boldsymbol{u}$ is a transformed control vector. The state space model then becomes

$$
\begin{aligned}
& \dot{\boldsymbol{z}}_{1}=\boldsymbol{z}_{2} \\
& \dot{\boldsymbol{z}}_{2}=\boldsymbol{u}
\end{aligned}
$$

Similarly, we can obtain an equivalent model in the task space: then we choose the state vector $\tilde{z}=\left[\tilde{z}_{1}^{T}, \tilde{z}_{2}^{T}\right]^{T}$, where $\tilde{z}_{1}=p$ and $\tilde{z}_{2}=\dot{p}$. From (4) we develop

$$
\ddot{\boldsymbol{p}}=J(q) \ddot{q}+J(q) \dot{q}
$$

Using (1) and (8) we find the control vector

$$
\tau=M(q) J^{-1}(q)[\tilde{u}-\dot{J}(q) \dot{q}]-n(q, \dot{q})
$$

which gives the linearized and decoupled system

$$
\ddot{\boldsymbol{p}}=\tilde{\boldsymbol{u}}
$$


and the state space model

$$
\begin{aligned}
& \dot{\tilde{z}}_{1}=\tilde{z}_{2} \\
& \dot{\tilde{z}}_{2}=\tilde{u}
\end{aligned}
$$

A proper controller for the double integrators (6) or (10), may easily be designed using linear control theory. In this paper linear quadratic optimal control is used.

\section{Augmentation of task space}

There is no unique transformation from a task space trajectory to a corresponding joint space trajectory for a redundant manipulator. The problem of tracking a task space reference trajectory with a redundant manipulator can be divided into two areas, first the kinematical problem of avoiding singularities and the loss of degrees of freedom, and second the control problem of following the trajectory without excessive input generalized forces.

\subsection{Kinematics}

In order to avoid singularities and the loss of degrees of freedom, we must be able to control the configuration of the redundant manipulator arm. For a nonredundant manipulator mounted on a positioning part, the configuration is given by the task space position $\boldsymbol{p}$ and the generalized coordinate $\tilde{\boldsymbol{p}}$ of the positioning part (Fig. 3).

We now define an augmented task space position vector

$$
p_{A}=\left[\begin{array}{l}
p \\
\tilde{p}
\end{array}\right]
$$

where $\operatorname{dim} \boldsymbol{p}_{\boldsymbol{A}}=\operatorname{dim} \boldsymbol{q}=\boldsymbol{n}$, and the elements of $\boldsymbol{p}_{\boldsymbol{A}}$ constiîute a set of generalized coordinates for the manipulator. The positional transformation can be expressed:

$$
p_{A}=h_{A}(q)
$$

Since $\operatorname{dim} p_{A}=\operatorname{dim} q$, there is an inverse transformation

$$
q=h_{A}^{-1}\left(p_{A}\right)
$$

There must be a finite number of solutions of (13), but when taking the joint limits into account usually only one solution remains. Based on (12) we define the aug-

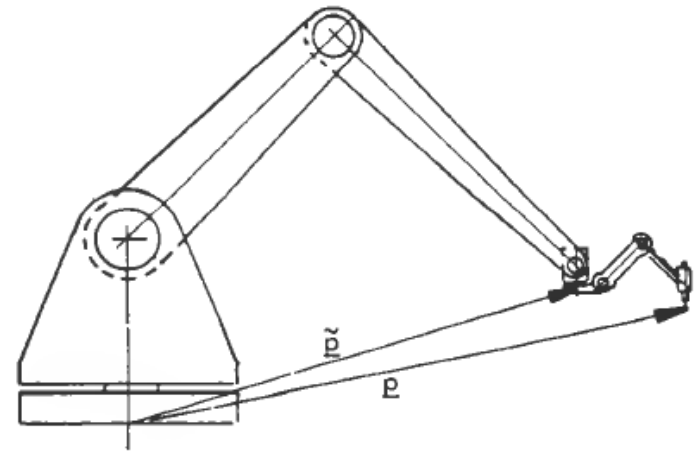

Figure 3. Augmented task space position vector of a redundant manipulator. 
mented Jacobian matrix

$$
J_{A}(q)=\frac{\partial p_{A}}{\partial q}
$$

which is a $n \times n$ matrix, and therefore invertible, except in singular points. This means that given the augmented task space velocity $\dot{p}_{A}$, we find the joint space velocity from

$$
\dot{q}=J_{A}^{-1}(q) \dot{p}_{A}
$$

From any given reference $\boldsymbol{p}_{\text {ref }}$, we now need to define a reference $\tilde{\boldsymbol{p}}_{\text {ref }}$ of the positioning part. This should be done so that singularities and the loss of degrees of freedom are avoided and so that the dynamical properties of this class of manipulators are utilized. An obvious choice of $\tilde{p}_{\text {ref }}$, will be one that gives the outer small manipulator a configuration with good manipulating abilities, i.e. close to the centre of its working area.

If the positioning part has three degrees of freedom and is used to give the base of the outer manipulator three transitional degrees of freedom, $\tilde{\boldsymbol{p}}_{\text {ref }}$ in many cases can be chosen as

$$
\tilde{p}_{\text {ref }}=d_{\text {ref }}-d_{0}
$$

Here $d_{\text {rer }}$ is the translational part of $p_{\text {ref }}$ and $d_{0}$ is a vector which is constant relative to the base of the outer non-redundant manipulator. $d_{0}$ is the translational position of the centre of the working area of the outer non-redundant manipulator with respect to its base. If the positioning part has one or two degrees of freedom, $\boldsymbol{p}_{\text {ref }}$ is chosen as a subset or a projection of (16); depending upon where the singular points of the positioning part are situated (Egeland 1987).

This choice of the reference $\tilde{p}_{\text {ref }}$ will, as long as $\boldsymbol{p}_{\text {ref }}$ is not approaching the limit of the working area of the manipulator, avoid singularities and the loss of degrees of freedom. However, $\tilde{\boldsymbol{p}}_{\text {ref }}$ can also be chosen in such a way that it minimizes a suitable performance index, e.g. the time used to complete the task.

\subsection{Control}

If an augmented task space reference $\boldsymbol{p}_{A, \text { ref }}$ is specified, a corresponding joint space reference $q_{\text {ref }}$ can be found from the inverse transformation (13). A controller may then be developed in the joint space.

However, for the particular class of redundant manipulators which is considered in this paper, there are serious drawbacks to this solution. If the hand is to be positioned accurately, all of the joint displacements $q_{i}, i=1, \ldots, n$, must be close to the corresponding references $q_{\text {ref, } i}$. This means that for a small, fast non-redundant manipulator mounted on a slow positioning part, the positioning part will limit the bandwidth of the system. This method of controlling the redundant manipulator will also require large input generalized forces in the joints. This is because high accelerations are required in the joints of the positioning part, and these joints move high inertias.

This drawback indicates that we should develop the controller in the task space. Even though we want to track the reference $\boldsymbol{p}_{\text {ref }}$ accurately, we can accept some deviation from the reference $\tilde{\boldsymbol{p}}_{\text {ref }}$ of the positioning part. This means that we should be able to give the redundant manipulator a high bandwidth and a large working 
area by distributing the high frequency motion to the outer, small manipulator, and the low frequency motion to the slow (and power consuming) positioning part.

The linearizing and decoupling control is found by (9):

$$
\tau=M(q) J_{A}^{-1}(q)\left[u-j_{A}(q) \dot{q}\right]-n(q, \dot{q})
$$

The state space model is then

$$
\begin{gathered}
\dot{x}_{1}=\boldsymbol{x}_{2} \\
\dot{\boldsymbol{x}}_{2}=\boldsymbol{u}
\end{gathered}
$$

where $x_{1}=p_{A}, x_{2}=\dot{p}_{A}$ and $u$ is the control vector transformed into the augmented task space.

We now develop a controller for the system (18) using linear quadratic optimal control theory (Athans and Falb 1966). The performance index is chosen as

$$
V=\lim _{T \rightarrow \infty} \frac{1}{T} \int_{0}^{T}\left(\Delta x^{T} Q \Delta x+u^{T} P u\right) d t
$$

where $x=\left[x_{1}^{T}, x_{2}^{T}\right]^{T}, \Delta x=x-x_{\text {ref }}, x_{\text {ref }}$ is the state reference, $Q=\operatorname{diag}\left(q_{11}, \ldots\right.$, $\left.q_{2 n, 2 n}\right)$ and $P=\operatorname{diag}\left(p_{11}, \ldots, p_{n n}\right)$.

High accuracy is desired for the tracking of the task space reference. Therefore, the weights $q_{i i}$ corresponding to the position deviations in task space should be high. The tracking of the reference for the positioning part need not be very accurate, and therefore the weights $q_{i i}$ corresponding to the position deviations of the positioning part are moderate. The resulting feedback control for the system (18) with the performance index given by (19) is then

$$
u_{i}=g_{i} \Delta x_{i}+g_{i+n} \Delta x_{i+n}+u_{0 i}
$$

where $g_{i}=-\sqrt{ }\left(q_{i i} / p_{i i}\right)$

$$
\begin{aligned}
& g_{i+n}=-\sqrt{ }\left(2 \sqrt{ }\left(q_{i i} / p_{i i}\right)+q_{i+n, i+n} / p_{i i}\right) \\
& u_{0 i}=\dot{x}_{\text {ref, } i+n}
\end{aligned}
$$

i.e. the last term, $u_{0 i}$, represents feedforward from the desired acceleration. Integral action may be included.

\section{Use of generalized inverses}

For a kinematically redundant manipulator the dimension of the joint space is greater than the dimension of the task space:

$$
\operatorname{dim}(\boldsymbol{q})=n>\operatorname{dim}(\boldsymbol{p})=m
$$

Consequently, the Jacobian matrix $J(q)$ is rectangular, and there is no unique inverse velocity transformation

$$
\dot{q}=J^{-1}(q) \dot{p}
$$

This is the underdetermined case.

A rectangular $n \times m$ matrix $A$ does not have an unique inverse $A^{-1}$, but it is possible to find a generalized inverse $B$ so that

$$
A B=I, \quad(n>m)
$$


In fact, there is an infinite number of matrices $B$ with the property (21).

The most commonly used generalized inverse is the pseudoinverse, or the Moore-Penrose inverse (Ben-Israel and Greville 1974). For $n>m$ this is

$$
J^{+}=J^{T}\left(J J^{T}\right)^{-1}
$$

This can easily be deduced by minimization of the criterion function

$$
H(\dot{\boldsymbol{q}})=\frac{1}{2} \dot{\boldsymbol{q}}^{T} Q \dot{\boldsymbol{q}}
$$

with $Q=I$, and subject to the constraint

$$
\dot{\boldsymbol{p}}=\boldsymbol{J}(\boldsymbol{q}) \dot{\boldsymbol{q}}
$$

i.e. use of a pseudoinverse corresponds to an instantaneous minimization of the (quadratic) joint velocities. The inverse transformation is then

$$
\dot{\boldsymbol{q}}=J^{+}(\boldsymbol{q}) \dot{\boldsymbol{p}}
$$

A general solution to (24) can be found by adding a null vector to the specific solution (25) (Klein and Huang 1983)

$$
\dot{\boldsymbol{q}}=J^{+}(q) \dot{p}+\left[I-J^{+}(q) J(q)\right] z
$$

An arbitrarily chosen vector $z$ is projected into the null space of $J(q)$, through the matrix $\left[I-J^{+} J\right]$. (Notice that adding the null vector $\dot{\boldsymbol{q}}_{n}=\left[I-J^{+}(q) J(q)\right] z$ does not affect the task space velocity vector $\dot{p}$ calculated by (24)). The common solution is then to choose $z$ to optimize a criterion $g(q)$. When maximizing we choose

$$
\boldsymbol{z}=\boldsymbol{\nabla} \boldsymbol{g}(\boldsymbol{q})
$$

The manipulability measure was introduced by Yoshikawa (1985a)

$$
w(q)=\sqrt{ } \operatorname{det}\left[J(q) J^{T}(q)\right]
$$

When $w(q)$ approaches the maximum value, the manipulator approaches a configuration with good kinematical properties, i.e. avoids singular configurations. We then choose

$$
z=\alpha \nabla w(q)
$$

where $\alpha$ is a positive gain constant.

Klein and Chirco (1987) investigated the criterion

$$
g(\boldsymbol{q})=\left(\boldsymbol{q}-\boldsymbol{q}_{\mathrm{c}}\right)^{T}\left(\boldsymbol{q}-\boldsymbol{q}_{\mathrm{c}}\right)
$$

where $\boldsymbol{q}_{\mathrm{c}}$ is a desired joint position, i.e. one specific configuration where the manipulator has good properties with respect to the avoidance of singularities etc. Other choices of the vector $z$ have been proposed (Klein and Huang 1983), (Liegeois 1977), but will not be considered here.

The solutions to the inversion problem have been based so far on pure kinematical considerations. If we want to take the dynamical characteristics of the manipulator into account, one approach is to choose the weight matrix $Q=M(q)$ in the criterion function (23), which now represents the kinematic energy of the systems. Minimization of (23) gives the generalized inverse

$$
J_{m}^{+}=M^{-1} J^{T}\left(J M^{-1} J^{T}\right)^{-1}
$$

$M(q)$ is the inertia matrix. 
However, in all cases the optimization is instantaneous, the opposite of optimal control theory where we consider system behaviour over a time interval. The effect of a generalized inverse in a control system is difficult to analyse, and may give non-optimal behaviour (as will be illustrated in the next section).

In the non-redundant case $(n=m)$ all generalized inverses reduce to the ordinary inverse $\left(J^{+}=J^{-1}\right)$. When the manipulator has too few degrees of freedom $(n<m)$, some tasks are impossible to perform; some configurations in the task space are impossible to realize. In this case there is an approximate generalized inverse similar to the pseudoinverse of the redundant case.

Let us turn to the controller design when using generalized inverses. According to the controller algorithm outlined in the previous section, we develop the controller in the task space. In $\S 4$ high and low frequency motion was distributed by decoupling in the augmented task space. In this case the joint motion will depend on the generalized inverse and the choice of null vector.

The control vector $\boldsymbol{u}$ might be found by optimal control as in the previous section, but in this case we only control the position of the end effector $p$.

We combine (9) and (26) into a task space linearizing and decoupling control

$$
\tau=M(q)\left\{J^{+}(q)[u-\dot{J}(q) \dot{q}]+\left[I-J^{+}(q) J(q)\right] z\right\}-m(q, \dot{q})
$$

where the pseudoinverse solution is obtained by choosing $z=0$. Now $u$ is the task space control vector of the $m$ decoupled double integrators.

In the non-redundant case $(n=m)$ the algorithms (17) and (31) both reduce to the resolved acceleration control of Luh et al. (1980).

\section{Simulation experiments}

In this section we will present a simple simulation experiment comparing the following three control algorithms presented in the previous sections:

(1) The augmented task space approach (17).

(2) The algorithm using the generalized inverse based on the manipulability measure, (28) and (31).

(3) The pseudoinverse algorithm, i.e. (31) with $z=\mathbf{0}$.

In the simulation experiments the idealized, planar, four-link manipulator shown in Fig. 4 was studied. The four joint angles are denoted by $q_{1}, q_{2}, q_{3}$ and $q_{4}$. The tip of the manipulator arm were to track a position reference in the horizontal $x y$-plane. For this task the manipulator was kinematically redundant.

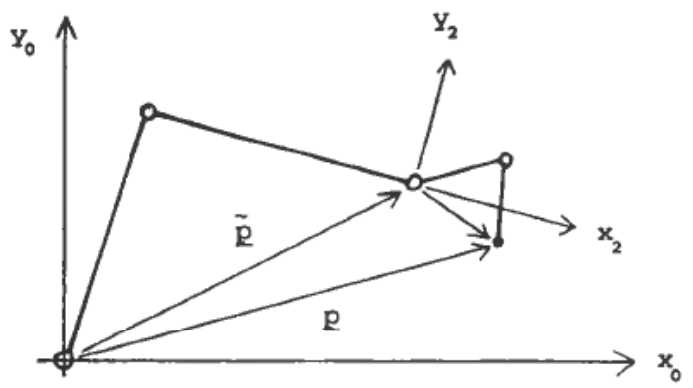

$$
\begin{array}{ll}
\mathrm{m}_{1}=10 \mathrm{~kg} & 1_{1}=1.0 \mathrm{~m} \\
\mathrm{~m}_{2}=10 \mathrm{~kg} & 1_{2}=1.0 \mathrm{~m} \\
\mathrm{~m}_{3}=2 \mathrm{~kg} & 1_{3}=0.2 \mathrm{~m} \\
\mathrm{~m}_{4}=2 \mathrm{~kg} & 1_{4}=0.2 \mathrm{~m}
\end{array}
$$

Figure 4. Planar manipulator. 


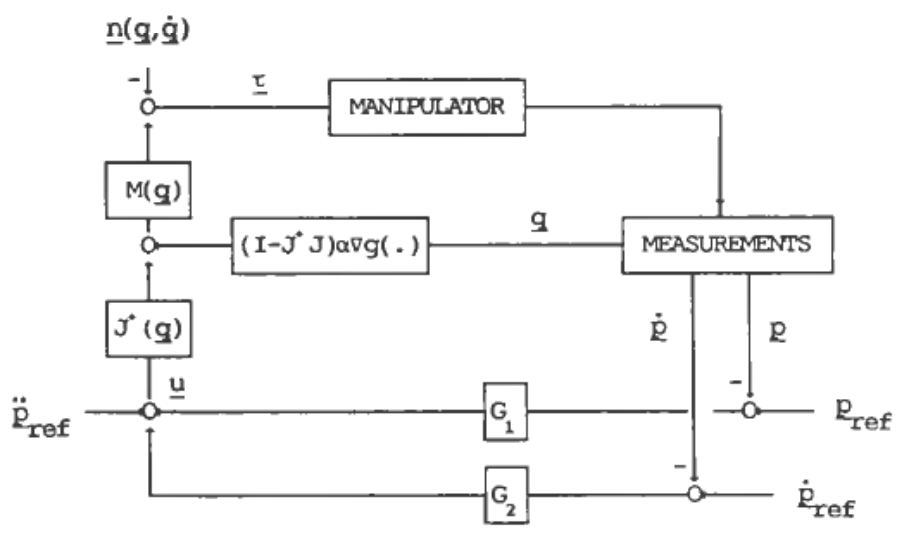

Figure 5. Controller, case 2.

Feedback linearization and decoupling in task space was used in all three cases. However, the term $\dot{J}(\boldsymbol{q}) \dot{\boldsymbol{q}}$ was not calculated as it was assumed to be negligible. The controllers for the decoupled double integrators were found by linear quadratic optimal control as given by (19) and (20).

The dimensions of the state vector $\boldsymbol{x}$ and the control vector $\boldsymbol{u}$ will be different in the augmented task space algorithm and in the generalized inverse algorithms. Therefore the weight matrices $P$ and $Q$ will also have varying dimensions, they were chosen as follows:

$$
\begin{aligned}
& \operatorname{dim} \boldsymbol{x}=8, \operatorname{dim} u=4 \\
& Q=\operatorname{diag}(10000,10000,100,100,200,200,2,2) \\
& P=\operatorname{diag}(1,1,10,10)
\end{aligned}
$$

(2) and (3) $\operatorname{dim} x=4, \operatorname{dim} u=2$

$$
\begin{aligned}
& Q=\operatorname{diag}(10000,10000,200,200) \\
& P=\operatorname{diag}(1,1)
\end{aligned}
$$

In case 2, the null vector gain $\alpha(28)$ was tuned to $\alpha=10$. The controller structure for case 2 is shown in Fig. 5, where feedforward from the desired acceleration is included. The controllers for cases 1 and 3 will be quite similar to this, the differences are given by (17) and (31) with $z=0$.

The augmented task space position vector $p_{A}=\left[p^{T}, \tilde{p}^{T}\right]^{T}$ was chosen as illustrated in Fig. 4. The two inner links constitute the positioning part of the manipulator, $\tilde{\boldsymbol{p}}$ is therefore the position of joint 3 . The reference $\boldsymbol{p}_{\text {ref }}$ was given, and the reference for the positioning part was calculated as

$$
\tilde{p}_{\text {ref }}=p_{\text {ref }}-C_{2}^{0} p_{0}^{2}
$$

where $p_{0}^{2}=[0 \cdot 2828,0]^{T}$, and $C_{2}^{0}$ is the rotational transformation matrix from coordinate system 2 to the base system 0 :

$$
C_{2}^{0}=\left[\begin{array}{lr}
\cos \left(q_{1}+q_{2}\right) & -\sin \left(q_{1}+q_{2}\right) \\
\sin \left(q_{1}+q_{2}\right) & \cos \left(q_{1}+q_{2}\right)
\end{array}\right]
$$

This will position the outer small manipulator in a configuration avoiding singularities. A zero velocity reference was used for the position part to give it a smooth movement. 

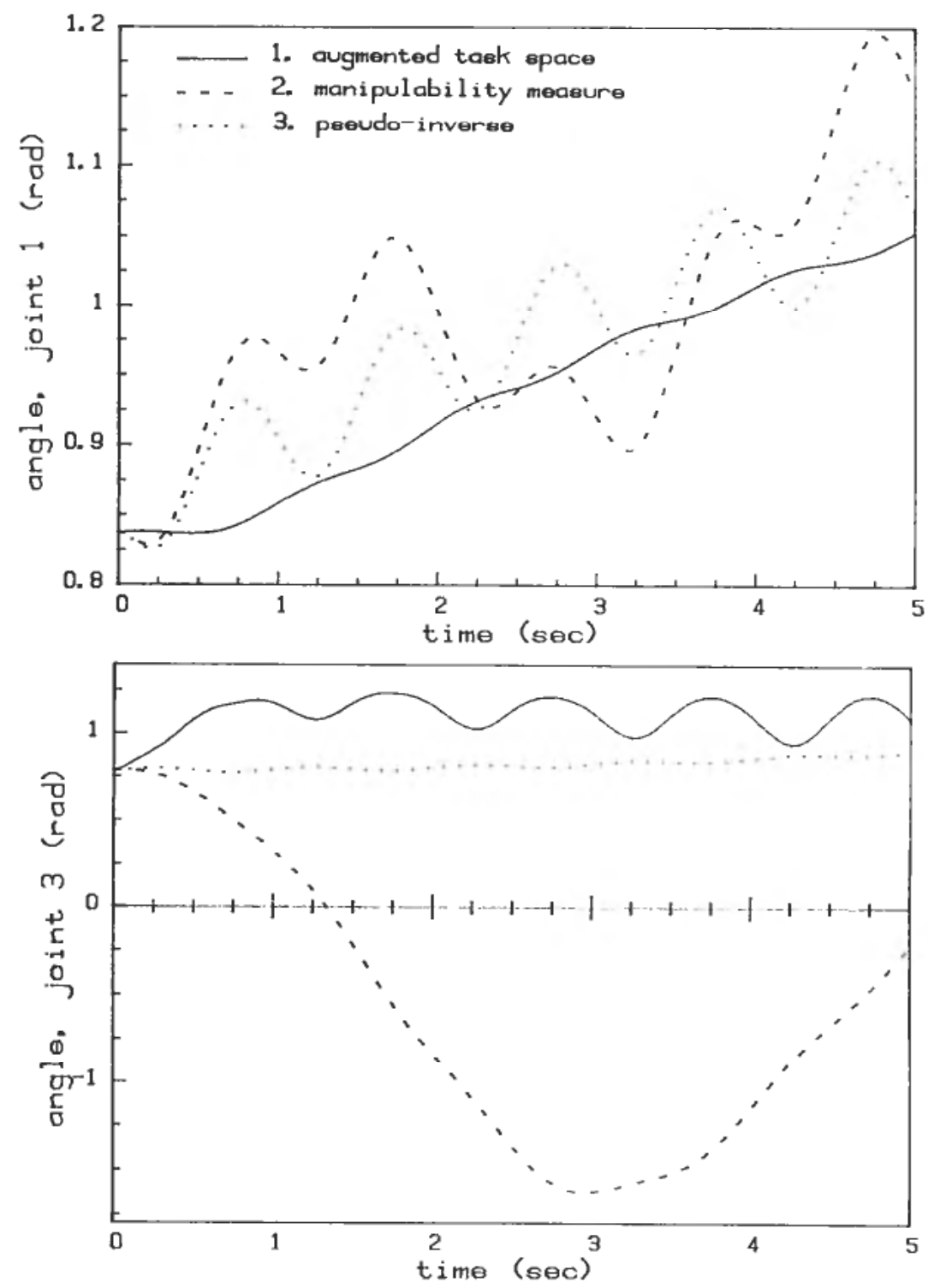

Figure 6. Joint angles.

In the simulation experiments the manipulator was to track a sinusoidal reference moving with constant velocity in the $y$-direction:

$$
p_{\text {ref }}(t)=\left[\begin{array}{c}
1.7+0.05 \sin 2 \pi t \\
0.1 t
\end{array}\right](m)
$$

Initially, the manipulator was at rest.

The simulation results are shown in Figs. 6,7 and 8. Fig. 6 shows the angles of joints 1 and 3 for the first 5 seconds of the simulation. Fig. 7 shows the torques of joints 1 and 3 . The responses of joints 2 and 4 are omitted because they are quite similar to the responses of joints 1 and 3 respectively.

It is clear from the results that the distribution of the high frequency motion to the outer joints and the low frequency motion to the inner joints functioned as 

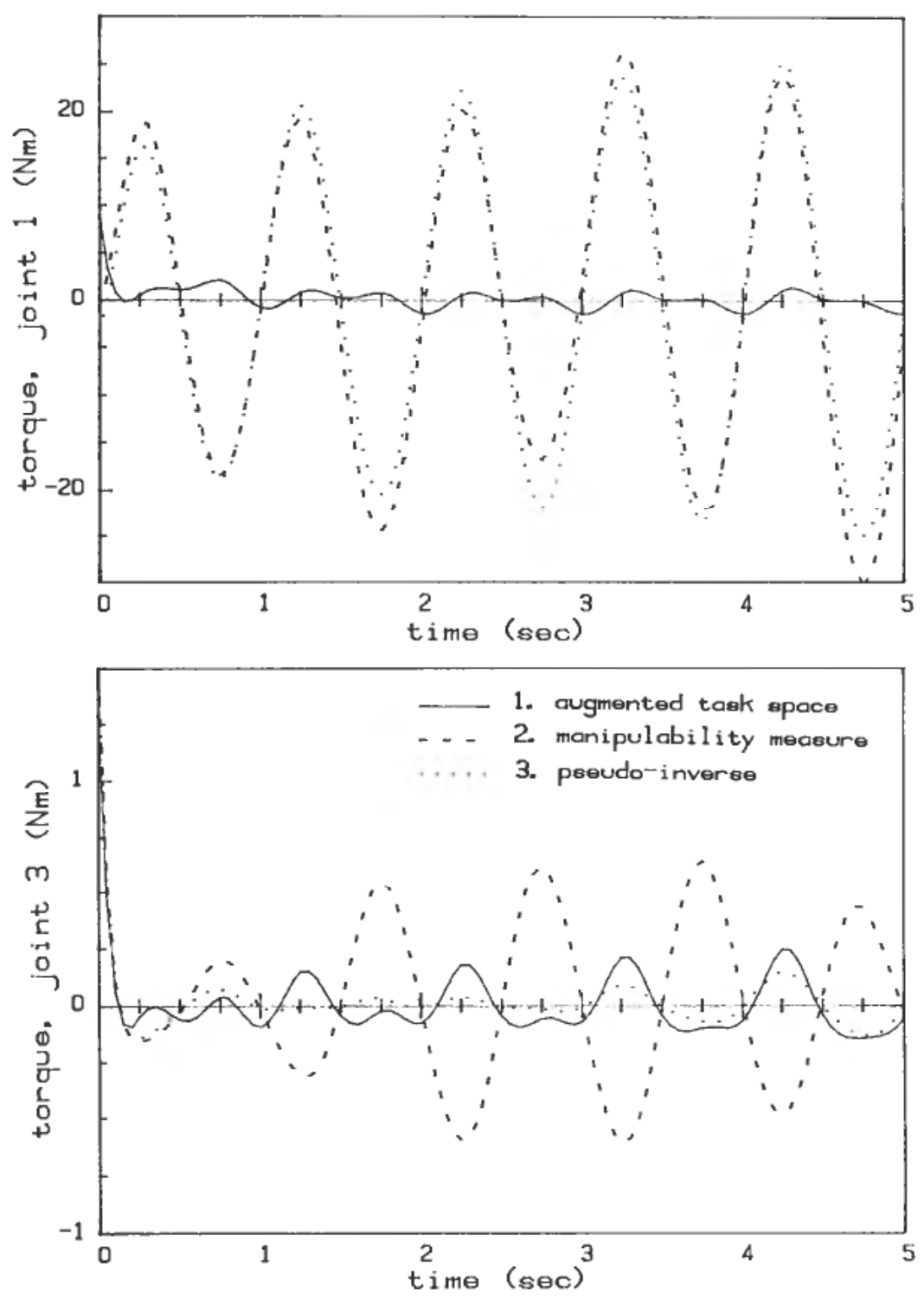

Figure 7. Joint torques.

proposed. For the augmented task space approach we see that joint 1 moved with almost constant speed and a very low actuator torque while joint 3 had a high amplitude sinusoidal motion with a moderate torque being applied to the joint.

With the pseudoinverse solution, case 3 , the situation is reversed. Now, both the fast and the slow motion was executed by the inner joints, while the two outer joints hardly moved at all. In fact, this is quite natural since the pseudoinverse minimizes the sum of the quadratic joint velocities: The inner joints will usually be situated farthest away from the tip of the manipulator arm, and will therefore realize any given task space velocity with lower joint velocities than the outer joints.

Case 2, the algorithm based on the maximization of the manipulability measure, gave a response analogous to the pseudoinverse. However, here the additional null space motion also gave an additional energy consumption, i.e. required higher 
torques. This null space motion optimizes a kinematical criterion function (27) without considering the manipulator dynamics: it forces the manipulator arm into a configuration which avoids singularities. In the current simulation example the manipulator was given an initial configuration far away from any singularlity so that in this example the increased energy consumption did not improve the tracking accuracy.

The difference in behaviour using the three algorithms can be illustrated by calculating the energy consumption

$$
E=\int_{0}^{5} \sum_{i}\left|\tau_{i} \dot{q}_{i}\right| d t
$$
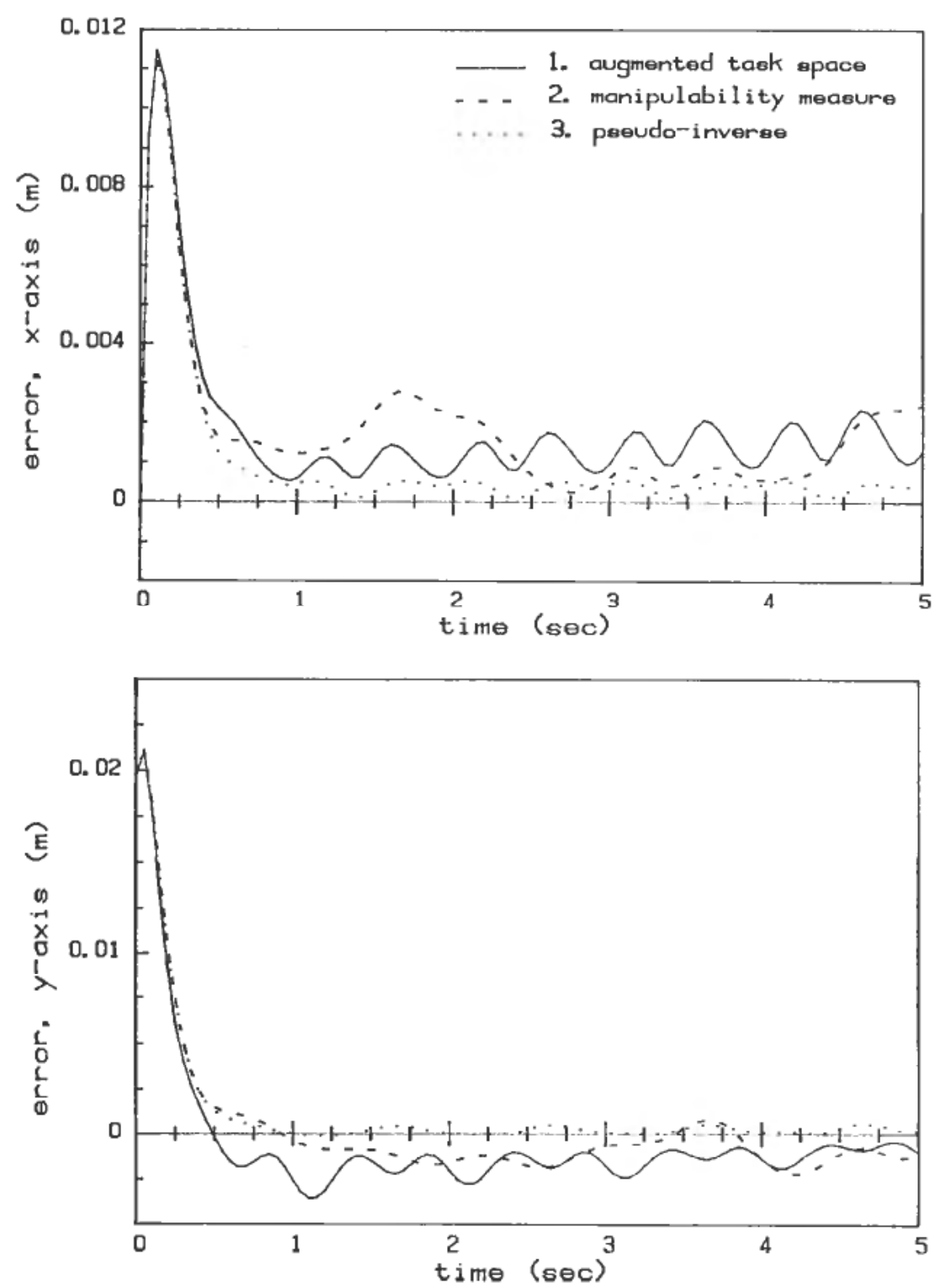

Figure 8. Tracking errors. 
which gave the following results

(1) $E=0.79 \mathrm{Ws}$

(2) $E=11 \cdot 1 \mathrm{Ws}$

(3) $E=13.4 \mathrm{Ws}$

Thus a reasonable proposition is: the better the control algorithm, the lower the energy consumption, provided of course that the tracking errors are of the same magnitudes.

Tracking errors in each of the three cases are shown in Fig. 8. The accuracy of the manipulability measure algorithm was clearly inferior to the pseudoinverse case, even though both seemed to be accurate within reasonable limits. The steady state error that occurred when using the augmented task space approach was probably due to the omission of the term

$$
\dot{J}(q) \dot{q}=\frac{\delta J(q)}{\delta q}(\dot{q})^{2}
$$

For any real manipulator the maximum actuator torques available will be limited. This means that when using the generalized inverse methods, a variety of tasks (i.e. high frequency references) will saturate the actuators of the inner joints, causing a considerable tracking error. Using the augmented task space approach the same tasks can be executed with good tracing accuracy and moderate energy consumption.

Notice that the preceding simulation experiments were carried out without deliberately favouring any properties of the different algorithms. It would have been quite simple to construct an example where the pseudoinverse was driven close to a singular point. The augmented task space algorithm would have avoided this singularity, and the differences between the responses would have become extremely large in favour of the latter.

\section{Conclusion}

An algorithm for dynamic control of redundant manipulators has been presented. The algorithm has been used for the control of manipulators consisting of a small non-redundant manipulator mounted on a larger positioning part. By a suitable choice of controller parameters, high and low frequency motion is distributed to the fast and slow parts of the manipulator. Singularities and the loss of degrees of freedom are avoided for simple manipulators by generating a reference for the positioning part on-line.

Even though the manipulators considered are restricted to a certain constructional class, we believe that the proposed control principle is fairly general because most redundant manipulators will have characteristics from this class. At least the inner links and actuators will always be dimensioned to carry the weight of the outer part of the arm.

Simulation experiments with a simple four-link planar manipulator showed that the control algorithm functioned as proposed. The experiments demonstrated that, for this class of manipulators, the augmented task space approach carried out the tasks with considerably lower energy consumption (i.e. lower torque for the inner joints) than the generalized inverse approaches. Also the bandwith of the system will be higher with this control algorithm. 
The investigation carried out by Klein and Chirco (1987) with the criterion (29), seems to confirm the drawbacks of the generalized inverse methods as demonstrated in this paper.

\section{ACKNOWLEDGMENT}

This work was supported by the Royal Norwegian Council for Scientific and Industrial Research under grant ED.0331.12510.

\section{REFERENCES}

Athans, M. and FalB, P. (1966). Optimal Control (McGraw-Hill, New York), pp. 750-813.

BAILLIEUL, J. (1985). Kinematic programming alternatives for redundant manipulators. Proc. IEEE Int. Conf. on Robotics and Automation, St. Louis, Missouri, pp. 722-728.

BEJCZY, A. K. (1974). Robot arm dynamics and control. JPL Technical Memo, 33-669.

Ben-ISRAEL, A. and Greville, T. N. E. (1974). Generalized Inverses: Theory and Applications (Wiley, New York), pp. 7-25.

EgELAND, O. (1987). Cartesian Control of Industrial Robots with Redundant Degrees of Freedom. Doctoral dissertation. The Norwegian Institute of Technology, Trondheim.

Hollerbach, J. M. and SuH, K. C. (1985). Redundancy resolution through torque optimization Proc. IEEE Int. Conf. on Robotics and Automation, St. Louis, Missouri, pp. 323-328.

Кнаттв, O. (1985). Real-time obstacle avoidance for manipulators and mobile robots. Proc. IEEE Int. Conf. on Robotics and Automation, St. Louis, Missouri, pp. 500-505.

Klein, C. A. and CHIRCO, A. I. (1987). Dynamic simulation of a kinematically redundant manipulator system. Journal of Robotic Systems, 4, 5-23.

Klein, C. A. and HuANG, C.-H. (1983). Review of pseudoinverse control for use with kinematically redundant manipulators. IEEE Trans. Systems, Man and Cybernetics, 13, $245-250$.

LIEGEOIS, A. (1977). Automatic supervisory control of the configuration and behaviour of multibody mechanisms. IEEE Trans. on Systems, Man and Cybernetics, 7, 868-871.

LUH, J. Y. S. (1983). Conventional controller design for industrial robots-a tutorial. IEEE Trans. Systems, Man and Cybernetics, 13, 298-316.

LuH, J. Y. S., WALKER, M. W. and PAuL, R. P. C. (1980). Resolved acceleration control of mechanical manipulators, IEEE Trans. Automatic Control, 25, 468-474.

LUNDE, E., EGELAND, O., BALCHEN, J. G. (1985). Cartesian control of a class of redundant robots. Preprints IF AC/IFIP/IMACS Int. Symposium on Theory of Robots. Vienna, pp. 101-106.

SalisburY, J. K. and Abramovitz, J. D. (1985). Design and control of a redundant mechanism for small motion. Proc. IEEE Int. Conf. on Robotics and Automation, St. Louis, Missouri, pp. 1016-1021.

Yoshikawa, T. (1985). Manipulability and redundancy of robotic mechanisms. Proc. IEEE Int. Conf. on Robotics and Automation, St. Louis, Missouri, pp. 1004-1009. 